\title{
Changes in Mesenchymal Cell and Hyaluronate Distribution Correlate with In Vivo Elevation of the Mouse Mesencephalic Neural Folds
}

\author{
JOYCE MORRIS-WIMAN AND LINDA L. BRINKLEY \\ Department of Anatomy and Cell Biology, Medical School, University of Michigan, \\ Ann Arbor, Michigan 48109
}

\begin{abstract}
The mesenchyme of the elevating mesencephalic neural folds of the mouse is composed primarily of mesenchymal cells embedded in an hyaluronate-rich extracellular matrix. In this study we provide evidence that hyaluronate and mesenchymal expansion may play a role in neural fold elevation and closure. Spatial and temporal patterns of mesenchymal cell and hyaluronate distribution were analyzed during neural fold elevation and closure using the computer-assisted method of smoothed spatial averaging and established methods of image processing. Degree of fold elevation and fold shape changes were analyzed using standard morphometric measures. The results of these analyses defined five distinct stages in mesencephalic neural fold elevation and closure. Mesenchymal cells and hyaluronate were found in a non-random distribution within the neural fold and showed distinct patterns of distribution which could be correlated with specific stages in neural fold elevation. The results of these analyses suggested that the elevation of the mesencephalic neural folds is produced by the expansion of an hyaluronate-rich extracellular matrix in the central mesenchyme which under the direction of surrounding tissues pushes the folds mediad towards the dorsal midline.
\end{abstract}

The process of neurulation, or the formation of the neural tube from a flat plate of cells, involves forces generated by some or all components of the neural fold. The identification of the forces involved and the tissue components that generate them has been the subject of research for over a century. In 1874, His first postulated that the forces behind neural fold elevation were generated within the neuroepithelium. Much of subsequent research has concentrated on the role of neuroepithelial cell shape change in neural fold elevation (for review, see Karfunkel, 1974). However, it now generally accepted that the interruption of the normal development of any of the components of the neural foldi.e., the surface ectoderm and the supporting mesenchyme, as well as the neuroepithelium-will result in the failure of the neural folds to elevate. Neural fold elevation may be driven in part by the stretching or flattening of the surface ectoderm (Schroeder, 1970; Brun and Garson, 1983; Schoenwolf and Fisher, 1983). It may also be produced by the directed expansion of the mesenchyme (Morriss and Solursh, 1978a,b; Schoenwolf and Fisher, 1983).

The mesenchymal compartment of the mesencephalic neural folds during their elevation is sparsely populated by cells embedded in an extracellular matrix (ECM) rich in glycosaminoglycan (GAG) (Solursh and Morriss, 1977; Morriss and Solursh, 1978a,b; Heifetz et al., 1980). Hyaluronate (HA), the predominant GAG during neural fold morphogenesis, has been shown to cause a high degree of hydration in experimental systems and has been associated with expanding intercel- lular spaces in other developing systems (for review, see Toole, 1981). It has been suggested that the expansion of the mesenchyme in fold morphogenesis may be the result of hydration of an HA-rich ECM (Solursh and Morriss, 1977; Morriss and Solursh, 1978a,b). If HA were uniformly distributed throughout the ECM, hydration of the matrix would result in an increase in the size of the neural fold, but not necessarily in fold elevation. Therefore, for the expansion of an HA-rich ECM in the mesenchyme to be an important driving force in neural fold elevation, HA must be non-randomly distributed throughout the fold and specific patterns of HA distribution must exist that change during the course of neural fold elevation. The localized expansion of the HA-rich ECM would tend to disperse resident mesenchymal cells. Thus changes in the degree of fold elevation would also be reflected in changes in patterns of mesenchymal cell distribution.

In this study we have identified five distinct stages of neural fold elevation using morphological observations and a series of simple morphometric measurements designed to evaluate changes in fold shape and degree of elevation. By employing established methods of image processing and image analysis, we have determined the patterns of mesenchymal cell and HA distribution during each of these stages. Changes in patterns of mesenchymal cell distribution were analyzed using the

\footnotetext{
Received April 26, 1989; accepted July 21, 1989.

Correspondence to: Joyce Morris-Wiman, University of Florida, College of Dentistry, Dept. of Orthodontics, Box J 444, J. Hillis Miller
} Health Center, Gainesville, FL 32610 
computer-assisted method of smoothed spatial averaging (Connelly and Bookstein, 1983; Brinkley and Bookstein, 1986) which allowed the identification of areas within the fold that have statistically significant differences in cell density. These differences were displayed as density contours. For the analysis of HA distribution, we used a computer-assisted method (Knoll et al., 1985; Brinkely and Morris-Wiman, 1987a,b) that allowed the registration and subtraction of Alcian blue-stained sections, one of which was digested by Streptomyces hyaluronidase, an enzyme specific for $\mathrm{HA}$ (Ohya and Kaneko, 1970). The resultant difference picture displayed the location of the HA in the neural fold. Our results indicate that major changes in the distribution of mesenchymal cells and HA occur which accompany fold expansion and elevation and that fold elevation is produced by the expansion of an HA-rich ECM in the mesenchyme.

\section{MATERIALS AND METHODS Animals}

Random-bred CD-1 mice were maintained in quarters with a dark cycle from 8:00 P.M. to 6:30 A.M. and fed Purina mouse chow and water ad libitum. Fertilization was assumed to occur between midnight and 2:00 A.M. of the morning a vaginal plug was found. Timed pregnant females (day0 0 plug date) were sacrificed by cervical dislocation at approximately $4 \mathrm{hr}$ intervals spanning the $24 \mathrm{hr}$ between gestational days 8 and 9 .

Each uterine horn was removed to a petri-dish containing Dulbecco's phosphate buffered saline where the uterine wall was opened with watchmaker's forceps and the decidua removed. Embryos were dissected from the decidua with membranes intact and examined under a dissecting microscope. The yolk sac diameter and the crown-rump length when applicable were measured and each embryo was assigned a morphological rating based on a modification of the scoring system of Brown and Fabrio (1981). We chose to group embryos according to their morphological rating because of the variability in the development of the cranial neural folds among embryos grouped according to somite number. The morphological ratings correspond to the somite numbers assigned by Jacobson and Tam (1982) as follows: MR3 corresponds to a 3 somite embryo; MR5 to 4-5 somites; MR7-MR9 to 6-8 somites; MR11-MR13 to $9-11$ somites; MR15 to $12-13$ somites, MR18 to $14-$ 17 somites; MR22 to $18-19$ somites.

\section{Analysis of Patterns of Mesenchymal Cell Distribution and Neural Fold Shape Changes}

\section{Histological techniques}

With the yolk sac and amnion removed the embryos were fixed for $2 \mathrm{hr}$ in $1 \%$ glutaraldehyde in $0.1 \mathrm{M}$ phosphate buffer, pH 7.2. They were then rinsed in buffer, dehydrated through graded alcohols, and embedded in glycol methacrylate (Sorvall). Two micron frontal sections from the mesencephalic region were Feulgenstained for morphometric analysis or toluidine bluestained for morphological observation.

\section{Data collection}

Two regions of the mesencephalic neural folds, one anterior and one posterior, were studied from a minimum of four different individuals taken from at least

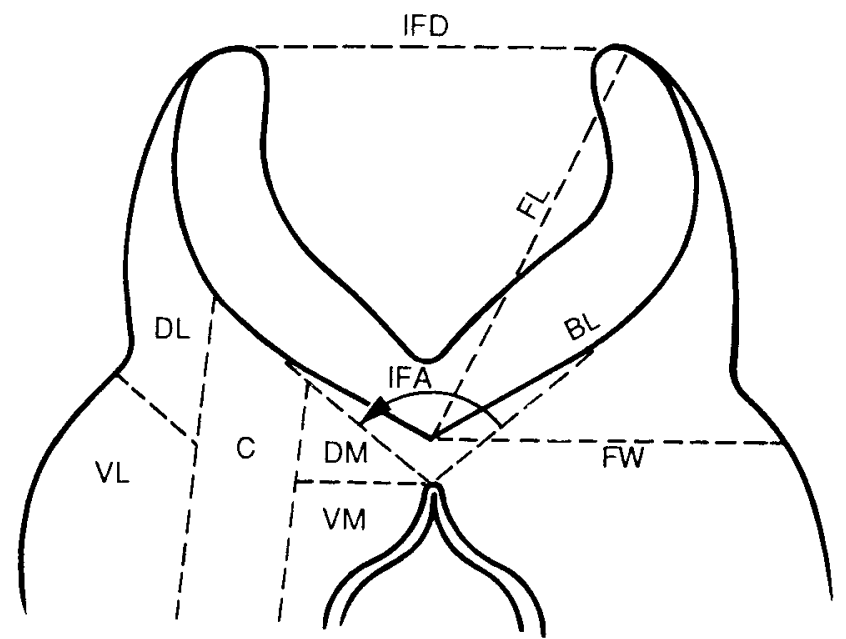

Fig. 1. IFD (interfold distance): The distance between the lateral edges of the two folds, defined as the point-to-point distance from the most lateral extent of the neuroepithelium of one fold to the most lateral extent of the neuroepithelium on the other. A decrease in this distance corresponds to an increase in the elevation and closure of the folds. FL (fold length): the distance from the most lateral point on the neuroepithelium to the midpoint of the neural groove. BL (basement membrane length): the length of the neuroepithelial basement membrane. FL/BL (the ratio between $\mathrm{FL}$ and $\mathrm{BL}$ ): if the dorsal surface of the fold is assumed to approximate a smooth curve-i.e., it is not scalloped-then as this ratio approaches 1 , the curvature of the fold decreases; as it deviates from 1 , the curvature increases. IFA (interfold angle): the angle formed by the intersection of two lines drawn tangent to the basal surface of the neuroepithelium at its midpoint along each fold. A decrease in this angle corresponds to an increased elevation of the folds. FW (fold width): the distance between the midpoint of the neural groove and the ventral extent of the squamous surface ectoderm. VL, ventrolateral fold; DL, dorsolateral fold; C, central fold; DM dorsomedial fold; VM ventromedial fold.

three different litters. For all parameters studied no significant differences were found between anterior and posterior regions or between right and left folds. The data reported represent the combined data from both regions and both folds. The mesenchymal compartment of each fold was delineated by the following boundaries: dorsally, by the basement membrane of the neuroepithelium; laterally, by the basement membrane of the surface ectoderm; medially, by a line extending from the neural groove to the basement membrane of the gut endoderm; and ventrally, by a line drawn from the lateral extent of the gut to the placode of the first arch. This compartment was further segmented into five regions: dorsolateral, ventrolateral, central, dorsomedial, ventromedial. (Fig. 1)

Using a Leitz Orthoplan microscope, photomicrographs were taken of complete frontal sections of each fold. A montage of photographic prints was produced at $\times 618$. From these photomontages the cross-sectional area was computed from an outline of the perimeter of the mesenchymal compartment using a Summagraphics digitizing tablet interfaced with a Tektronix 4054 computer running a simple micrograph measurement program. Also measured were the following: the pointto-point distance between the lateral edge of the neuroepithelium and the midpoint of the neural groove (fold length, FL); the point-to-point distance between the lateral edge of the neuroepithelium of one fold and 
the other (interfold distance, IFD); the length of the neuroepithelial basement membrane (basement membrane length, BL); the point-to-point distance between the midpoint of the neural groove and the ventral extent of squamous surface ectoderm (fold width, FW); and the angle formed by the intersection of two lines drawn tangent to the basal surface of the neuroepithelium at its midpoint in each fold (interfold angle, IFA) (Fig. 1).

\section{Smoothed spatial averages}

The nuclei of mesenchymal and neural crest cells contained within the mesenchymal compartment were located in the photomontages of each fold and digitized together with a string of points representing the fold outline. These data were analyzed using a series of programs described previously (Connelly and Bookstein, 1983; Brinkley and Bookstein, 1986) and briefly reviewed here. A rectangular grid of smoothing boxes was placed over the image of each section and the number of cells was determined for each grid box. The number of cells per $600 \mu^{2}$ was then computed for each grid box. These values were linearly interpolated between adjacent smoothing box centers and converted to contour maps over the interior of the section. These contour maps were then gray level coded to aid in discrimination of patterns.

\section{Analysis of Patterns of Hyaluronate Distribution}

\section{Histological techniques}

Embryos were fixed for $4 \mathrm{hr}$ in $4 \%$ paraformaldehyde in $0.1 \mathrm{M}$ phosphate buffer, $\mathrm{pH} 7.2$, with $0.5 \%$ cetylpyridinium chloride (CPC) added to precipitate glycosaminoglycans (Derby and Pintar, 1978). They were then dehydrated through graded alcohols, cleared in methyl benzoate, and embedded in paraffin. Alternate $7 \mu \mathrm{m}$ frontal sections of the mesencephalic region were incubated either in $100 \mathrm{TRU} / \mathrm{ml}$ Streptomyces hyaluronidase (SH) in $0.1 \mathrm{M}$ sodium acetate buffer ( $\mathrm{pH} 5.0$ ) in $1 \mathrm{U} / \mathrm{ml}$ chondroitinase ABC in Tris-HCL buffer ( $\mathrm{pH}$ 8.6 ), or in buffer alone. Sections were then stained overnight in $1 \%$ Alcian blue 8GX (Mallinkrodt, Paris, $\mathrm{KY}$ ) in $3 \%$ acetic acid ( $\mathrm{pH} 2.6$ ) with $0.025 \mathrm{M} \mathrm{MgCl}_{2}$, dehydrated in graded ethanols, cleared in xylene, and mounted in permount.

\section{Image processing}

Streptomyces hyaluronidase-digested and adjacent control sections were viewed and photographed with a Leitz Orthoplan photomicroscope. A $603 \pm 10 \mathrm{~nm}$ filter was placed in the light path to intensify visualization of the Alcian blue stain. The negatives were digitized using an Optronics rotating drum scanner with a spot size of $50 \mu \mathrm{m}$. The images were recorded as $512 \times 482$ pixel matrices of brightness values of 0 (black) to 255 (white).

The images were analyzed using a computer-assisted method described previously (Knoll et al., 1985; Brinkley and Morris-Wiman, 1987a,b). Using an International Imaging Systems Model 75 (IIS, San Jose, CA) image processor linked to a MassComp MC-500 computer (Westford, MA), the digested and undigested images were subtracted from one another to produce an image with gray values representing only stained material which was removed by Streptomyces hyaluroni- dase-digestion, i.e., HA. Because paraffin sections tend to expand unevenly when applied to subbed microscope slides, even consecutive sections do not register with one another well enough to allow their subtraction. The IIS has an indwelling warper which was used to fit the digested image to the undigested. The two images were then subtracted from each other to produce a difference picture image (DPI). The histogram of each DPI was then matched, or mapped into a standard histogram which had a median gray value of 128 and a standard deviation of 27.5. The gray values of the background and cells were found to be above 150. Therefore all gray values above 150 were converted to 255 . Thus an image was produced which contained gray values that corresponded only to Alcian blue-stained HA. These images were used as the input images in a thresholding function that segments the range of gray values into six steps. Each step of increasing density represents an increase in the relative concentration of $\mathrm{HA}$. In these final images the boundaries between gray levels were smoothed using a median filter of $5 \times 5$ matrix.

\section{RESULTS \\ Morphological Changes During Neural Fold Elevation (Fig. 2)}

At MR3 and MR5 the mesencephalic neural folds are small and biconvex, encased laterally and dorsally by a columnar epithelium. There is a slight decrease in the height of the dorsal neuroepithelium (NE) in the medial regions of the fold, contributing to the splayed appearance of the folds. The dorsal NE curls around the dorsolateral fold to meet the adjacent surface ectoderm (S). There is as yet no clear demarcation between neuroectoderm and surface ectoderm. Mesenchymal cells are distributed throughout the fold except in the fold region adjacent to the most medial portion of the neural groove (hereafter, referred to as the neural groove) which is cell-free.

The neural folds at MR7 appear to have increased in height and width. The NE in central and lateral portions of the folds has thickened through an increase in the height of its cells and through a change from columnar to pseudostratified organization. The NE forming the neural groove remains columnar, but the cells are wedge-shaped. The dorsolateral edge of the neuroepithelium no longer is curled ventrally to meet its adjacent $\mathrm{S}$ and the decrease in the height of surface ectoderm cells makes the junction between $\mathrm{NE}$ and $\mathrm{S}$ very distinct. The mesenchymal compartment of the fold is more cellular than at earlier stages. This is particularly true in the lateral portions of the fold, which are populated with large, rounded, migrating neural crest cells. The medial portions of the fold adjacent to the neural groove remain cell-free although mesenchymal cells extend further into the area bounded by the neural groove.

At MR9 the folds are characteristically planar from the convex rim of the neural groove to the NE-S interface. Over the planar portion of the fold the pseudostratified NE has increased in height. The neural groove, lined by a columnar $\mathrm{NE}$ with wedge-shaped cells, intersects the flattened body of the fold at an almost right angle. The flattening of the folds is concomitant with the slight lateral stretching of the S ad- 

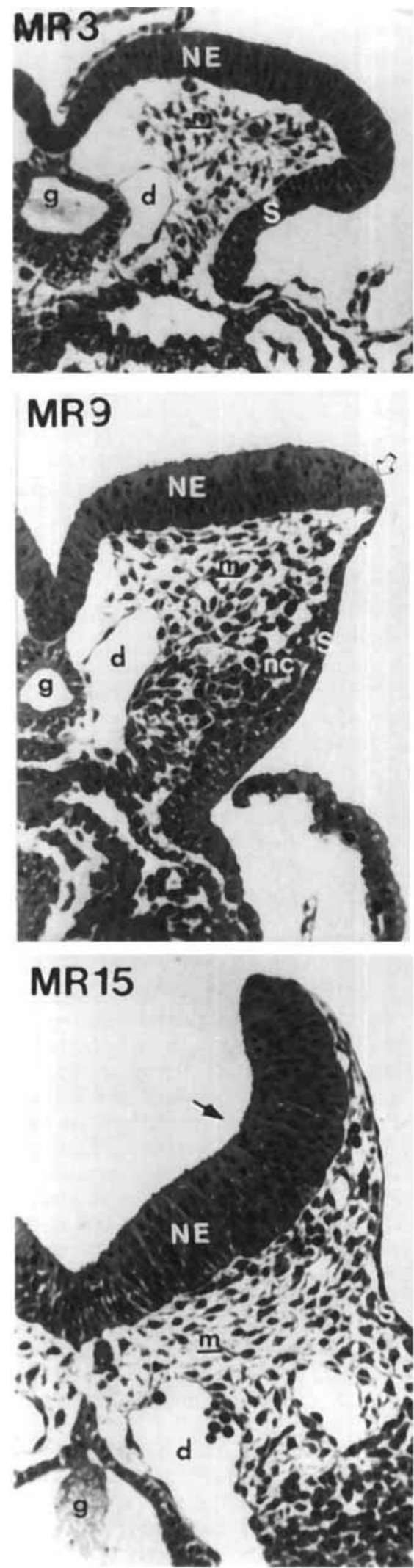
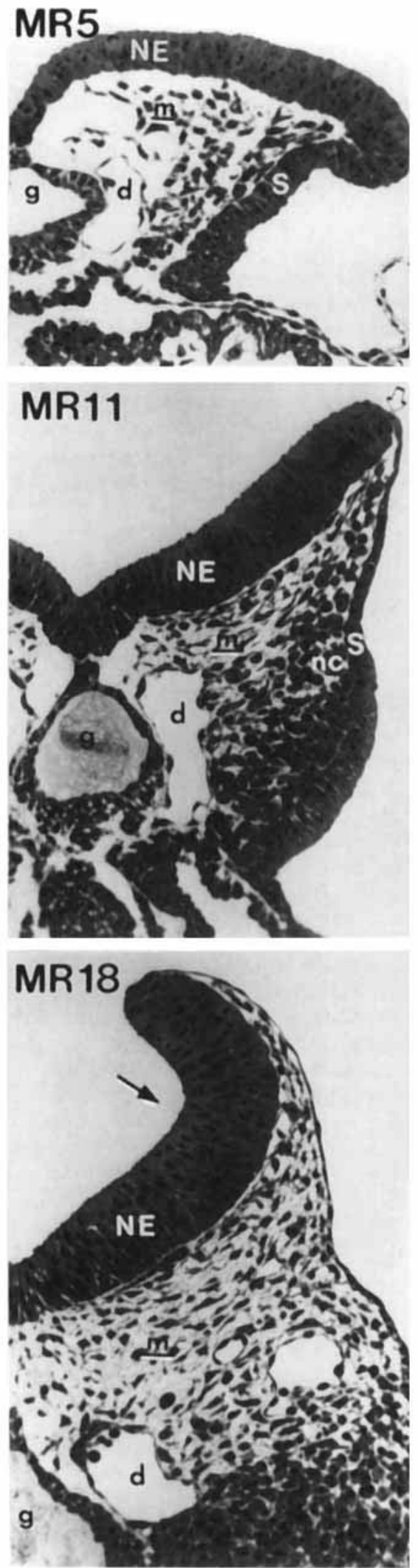
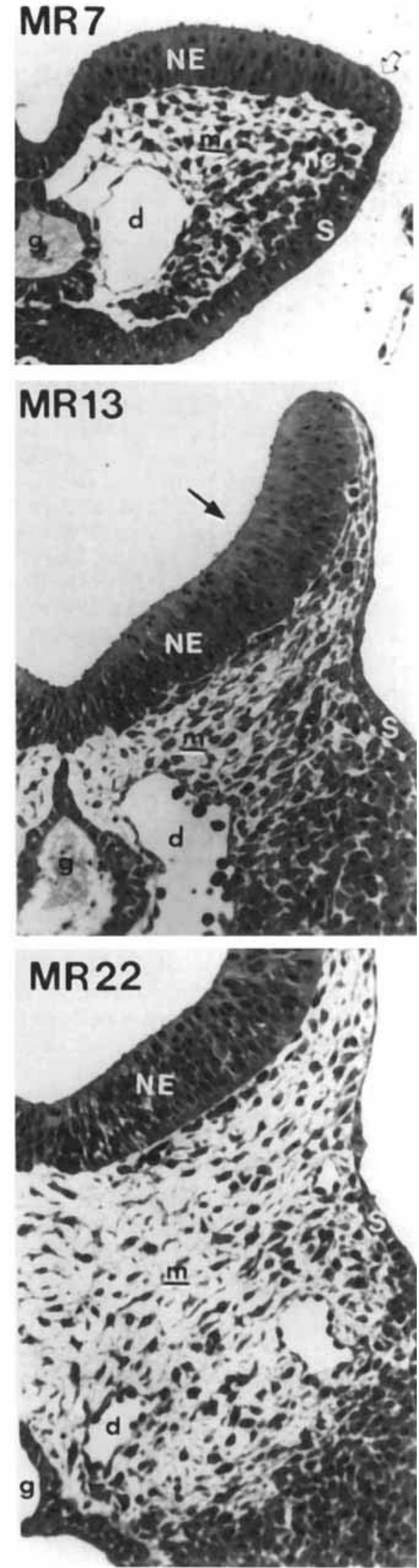

Fig. 2. 
TABLE 1a. Changes in parameters of fold shape during elevation and convergence of the mesencephalic neural folds ${ }^{1}$

\begin{tabular}{|c|c|c|c|c|c|c|c|c|}
\hline Stage & $\mathbf{N}^{2}$ & $\begin{array}{r}\mathbf{F} \\
\text { len } \\
(\end{array}$ & $\begin{array}{l}\text { old } \\
\text { gth } \\
\text { im) }\end{array}$ & $\begin{array}{c}\text { Basement } \\
\text { membrane } \\
\text { length } \\
(\mu \mathrm{m})\end{array}$ & Ratio $^{4}$ & $\begin{array}{l}\text { Interfold } \\
\text { distance } \\
(\mu \mathrm{m})\end{array}$ & $\begin{array}{c}\text { Interfold } \\
\text { angle } \\
\text { (degrees) }\end{array}$ & $\begin{array}{c}\text { Fold } \\
\text { width } \\
(\mu \mathrm{m})\end{array}$ \\
\hline 3 & 6 & 229.55 & \pm 24.30 & $288.37 \pm 46.63$ & $0.80 \pm 0.06$ & - & - & $192.40 \pm 20.36$ \\
\hline 5 & 4 & 281.04 & $\pm 37.21^{*}$ & $363.91 \pm 47.92$ & $0.77 \pm 0.06$ & 一 & 一 & $236.28^{*} \pm 35.40$ \\
\hline 7 & 7 & $303.15^{*}$ & \pm 27.50 & $365.75^{*} \pm 41.08$ & $0.83^{*} \pm 0.04$ & 一 & - & $277.45^{*} \pm 25.39$ \\
\hline 9 & 8 & $342.35^{*}$ & \pm 22.50 & $383.84 \pm 22.82$ & $0.89^{*} \pm 0.03$ & $632.12 \pm 53.57$ & $150.29 \pm 15.18$ & $304.68^{*} \pm 21.94$ \\
\hline 11 & 8 & 351.98 & \pm 20.56 & $388.98 \pm 26.81$ & $0.91 \pm 0.04$ & $596.39 \pm 41.25$ & $131.34^{*} \pm 11.58$ & $284.63^{*} \pm 19.79$ \\
\hline 13 & 6 & $374.57^{*}$ & \pm 20.46 & $427.14^{*} \pm 35.25$ & $0.88^{*} \pm 0.04$ & $553.11^{*} \pm 73.61$ & $132.05 \pm 11.19$ & $263.78^{*} \pm 23.37$ \\
\hline 15 & 5 & 387.42 & \pm 31.08 & $432.27 \pm 38.69$ & $0.90 \pm 0.03$ & $491.19 * \pm 46.49$ & $102.35^{*} \pm 20.07$ & $274.73 \pm 23.28$ \\
\hline 18 & 8 & 377.77 & \pm 33.28 & $492.59^{*} \pm 36.96$ & $0.77^{*} \pm 0.07$ & $309.04 * \pm 54.83$ & $94.42 \pm 6.08$ & $262.98 \pm 27.52$ \\
\hline 22 & 5 & 396.04 & \pm 40.32 & $645.57^{*} \pm 70.09$ & $0.62 * \pm 0.04$ & $0^{*}$ & $86.70 \pm 11.41$ & $259.02 \pm 30.15$ \\
\hline
\end{tabular}

${ }^{1}$ Values given are the mean \pm the standard deviation.

${ }^{2} \mathrm{~N}$, No. of embryos examined.

${ }^{3}$ For explanation of measurements, see Figure 1.

4The ratio of the fold length to the basement membrane length.

*Mean is significantly different $(P \leq 0.01)$ from that of the preceding stage by analysis of variance.

joining the lateral NE to enclose a distinct dorsolateral mesenchymal compartment. This region may be cellfree or contain newly emerged neural crest cells. The medial mesenchyme adjacent to the neural groove remains cell-free. Mesenchymal cells do not penetrate the region delineated by the rim of the neural groove, the neural groove, the dorsal aorta, and the gut and notochord.

At MR11 the planar folds are elevated and V-shaped. The elevation is concomitant with an increase in the dorsolateral mesenchymal region enclosed by squamous $\mathrm{S}$ and the lateral $\mathrm{NE}$. In the central fold, mesenchymal cells have undergone a pronounced reorganization showing a definite preference for an orientation parallel to the transverse plane of the NE. The medial mesenchyme remains cell-free; the lateral mesenchyme is still populated by migrating neural crest cells.

The further elevation of the neural folds at MR13 is concomitant with the further increase in the dorsolateral edge and with the appearance of lateral furrows populated by wedge-shaped cells, resulting in a slight mediad curvature. The NE has increased in height except in the neural groove which is lined by wedgeshaped NE cells. Mesenchymal cells have invaded the formerly cell-free region adjacent to the neural groove.

The elevation of the folds at MR15 is also concomitant with the lengthening and narrowing of the dorsolateral fold, but an increase in the mediad curvature is not apparent. Instead the elevation appears to be the result of a decrease in the angle between opposite folds at the neural groove. There is a large increase in the size of the fold at this stage. In medial and central portions of the fold, mesenchymal cells are more widely separated than previously and medially they may appear randomly oriented.

Fig. 2. Toluidine blue-stained frontal sections of the mesencephalic neural folds during neural fold elevation and closure. NE, neuroepi thelium; S, surface ectoderm; m, mesenchyme; nc, neural crest; arrow lateral furrow; open arrow, NE/S junction; $g$, gut; $d$, dorsal artery. $\times 166$.
At MR18 the lateral edges of the fold approach each other, or converge, as the mediad curvature of the lateral NE appears to increase. The folds are now Cshaped with a shallow neural groove. The expanded central and medial regions of the fold are sparsely populated by mesenchyme cells which are widely separated and appear randomly oriented. Often it is only at the base of the elevated dorsolateral fold that mesenchymal cells appear to retain their orientation to the neuroepithelial basement membrane. The neural groove has become detached from the notochord and foregut, allowing mesenchymal cells to encroach on the region dividing the folds. Blood vessels subjacent to the neuroepithelium are expanded and may interconnect with one another.

At MR22 the neural folds have fused to form the neural tube proper. The mesenchymal compartment has undergone considerable dorsoventral expansion. As a result the ventral portion of the neural tube, the former neural groove, is widely separated from the notochord and gut. Randomly oriented mesenchymal cells are now widely dispersed throughout this medial region and the central regions of the mesenchyme. Only in the dorsolateral fold do mesenchymal cells show some alignment with the neuroepithelial layer.

\section{Changes in Fold Shape During Fold Elevation}

Changes in fold shape and degree of elevation were evaluated using the following parameters: interfold distance, or the distance between the lateral edges of opposite folds, as a measure of the degree of fold closure; fold length, or the distance from the lateral edge of a fold to the midpoint of the neural groove; neuroepithelial basement membrane length; the ratio of fold length to basement membrane length, as a measure of the degree of fold curvature; interfold angle, or the angle between opposite folds at the neural groove, as a measure of fold elevation; and fold lateral width, as a measure of mesenchymal lateral expansion. (Fig. 1; Table 1a)

From MR5 to MR11 the length of the basement membrane does not change, but the fold length significantly increases between stages. From MR15 to MR22 the fold 
TABLE 1b. Changes in mesenchymal cell density and fold cross-sectional area ${ }^{1}$

\begin{tabular}{ccccc}
\hline Stage & $\mathrm{N}^{2}$ & $\begin{array}{c}\text { Area } \\
\left(10^{4} \mu \mathrm{m}^{2}\right)\end{array}$ & $\begin{array}{c}\text { No. of cells } \\
\text { per fold }\end{array}$ & $\begin{array}{c}\text { Cell density } \\
\left(\text { cells } / 600 \mu \mathrm{m}^{2}\right)\end{array}$ \\
\hline 3 & 6 & $\mathbf{1 . 5 1} \pm 0.37$ & $48.00 \pm 10.48$ & $1.75 \pm 0.45$ \\
5 & 4 & $2.56 \pm 0.53^{*}$ & $72.57 \pm 19.67^{*}$ & $1.61 \pm 0.26$ \\
7 & 7 & $3.53 \pm 0.45^{*}$ & $123.23 \pm 20.07^{*}$ & $1.97 \pm 0.31^{*}$ \\
9 & 8 & $3.49 \pm 0.37$ & $122.17 \pm 10.20$ & $2.02 \pm 0.20$ \\
11 & 8 & $3.68 \pm 0.41$ & $145.52 \pm 17.42^{*}$ & $2.29 \pm 0.24^{*}$ \\
13 & 6 & $3.65 \pm 0.83$ & $149.92 \pm 27.14$ & $2.46 \pm 0.27$ \\
15 & 5 & $4.51 \pm 0.76^{*}$ & $164.20 \pm 13.81^{*}$ & $2.15 \pm 0.37^{*}$ \\
18 & 8 & $5.50 \pm 0.69^{*}$ & $198.98 \pm 33.13^{*}$ & $2.13 \pm 0.43$ \\
22 & 5 & $9.73 \pm 0.20^{*}$ & $287.40 \pm 48.58^{*}$ & $1.84 \pm 0.28^{*}$ \\
\hline
\end{tabular}

${ }^{1}$ Values given are the mean \pm the standard deviation.

${ }^{2} \mathrm{~N}$, No. of embryos examined.

*Mean is significantly different $(P \leq 0.01)$ from that of preceding stage by analysis of variance.

length remains constant, whereas the basement membrane length increases significantly with each stage. The ratio of the fold length to basement membrane length increases at MR9 when the folds become planar to decrease in curvature. The ratio decreases at MR13 with the acquisition of the ventral flexure. The decrease in the interfold distance between MR13 and MR15 is not accompanied by a significant increase in the curvature of the fold, or by a significant decrease in the fold length to basement membrane length ratio. However, it is concomitant with a significant decrease in interfold angle indicating increased elevation of the folds. At MR18 and MR22 the curvature increases (the fold length to basement membrane length ratio decreases) as the interfold distance decreases, but there is no significant change in interfold angle. The lateral fold width increases significantly between all stages from MR3 to MR9. It decreases at MR11 and again at MR13 and then does not change significantly throughout the remainder of neural fold elevation.

\section{Changes in Mesenchymal Cell Density and Mesenchymal Cross-Sectional Area During Neural Fold Elevation}

The overall mesenchymal cell density, expressed as the number of cells per $600 \mu \mathrm{m}^{2}$, was computed for each fold studied. The total area of the mesenchymal compartment and the total number of mesenchymal cells in the fold were also evaluated at each stage (Table 1b).

The cross-sectional area of the fold mesenchyme increases significantly from MR3 to MR7 and then remains unchanged until MR15 as the folds flatten and acquire lateral furrows. It increases significantly between subsequent stages MR15, MR18, and MR22. Increases in overall cell density between MR5 and MR7 and between MR9 and MR11. A decrease occurs between MR13 and MR15 and again between MR18 and MR22.

\section{Changes in Mesenchymal Cell Distribution During Neural Fold Elevation}

The computer-assisted method of smoothed spatial averaging allows the viewer to assess qualitatively the quantitative analysis of patterns of mesenchymal cell distribution within a neural fold. The smoothed spatial averages are plotted as density contour maps representing statistically significant differences in cell den- sity within a particular fold. However, no method as yet exists to compare plots from different folds statistically. Therefore, the contour plots of different folds must be visually compared by the investigator. Plots of smoothed spatial averages were examined to ascertain if the changes in cell density occur throughout the fold or if these changes are localized to specific regions of the fold. The gray level coded plots seen in Figure 3 are representative of each morphological rating studied. At all stages the lateral regions of the fold are more densely populated. At MR3 and MR5 a ventrolateral pocket of high cell density is established. The majority of the cells populating these regions are newly emerged neural crest cells. At MR11 a definitive dorsolateral elevating edge is observed and a small pocket of high cell density is established in its base. Lateral to this area of increased cell concentration the fold's elevating edge is relatively cell-free. In the later stages of neural fold elevation this pattern is maintained and the region of increased cell concentration appears to underlie the lateral furrows in the NE.

The most medial regions of the neural fold are relatively cell-free at all stages. The dorsal aorta occupies a major portion of the ventromedial mesenchyme, thus explaining the dearth of cells in this area. The central fold at MR3 and MR5 is well populated, but the expansion of the fold at MR7 is accompanied by a decrease in cell density in both the central and medial fold. Only a small pocket of high cell density in the dorsomedial fold lateral to the rim of the neural groove remains. At MR9 and MR11 this pocket is expanded into a band of increased cell density which extends on a diagonal from the dorsomedial to the ventrolateral fold. At MR13 all regions of the fold show an increase in cell density, but the relative increase is greater in the central and medial fold regions. The band of increased cell density is shifted slightly laterally.

The expansion of the fold at MR15 and MR18 is concomitant with a decrease in cell density, particularly in the medial and central fold regions. The band of increased cell density extending through the central fold is maintained at these stages. At MR22 the central and medial regions are sparsely populated. Only two pockets of increased cell density exist. One pocket corresponds to the ventrolateral fold which is the dorsal extent of the first arch. The other is in the dorsolateral fold beneath the thinned elevated edge.

\section{Changes in Patterns of Hyaluronate Distribution (Fig. 4)}

The images described below are difference picture images (DPI) in which digitized images of Streptomyces hyaluronidase-digested, Alcian blue-stained sections of neural folds were subtracted from adjacent, undigested Alcian blue-stained sections. Streptomyces hyaluronidase is an enzyme that specifically degrades HA (Ohya and Kaneko, 1970). Therefore, the resultant difference pictures would depict the distribution of HA. However, in the mesenchyme of the early embryos, HA may be complexed with other GAGs and glycoproteins in such a way that its digestion may lead to the extraction of these other matrix components (see Fisher and Solursh, 1977; Morriss and Solursh, 1978a; MorrissKay et al., 1986). For several embryos at different stages of neural fold elevation, sections which were adjacent to undigested, buffer-incubated control sections 


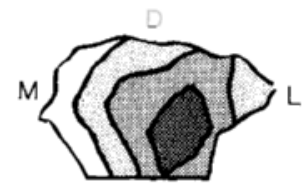

MR 3

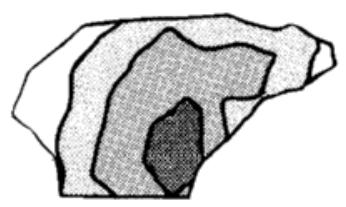

MR 5

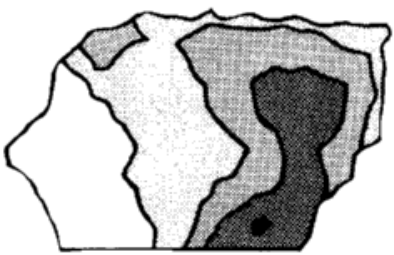

M R 7
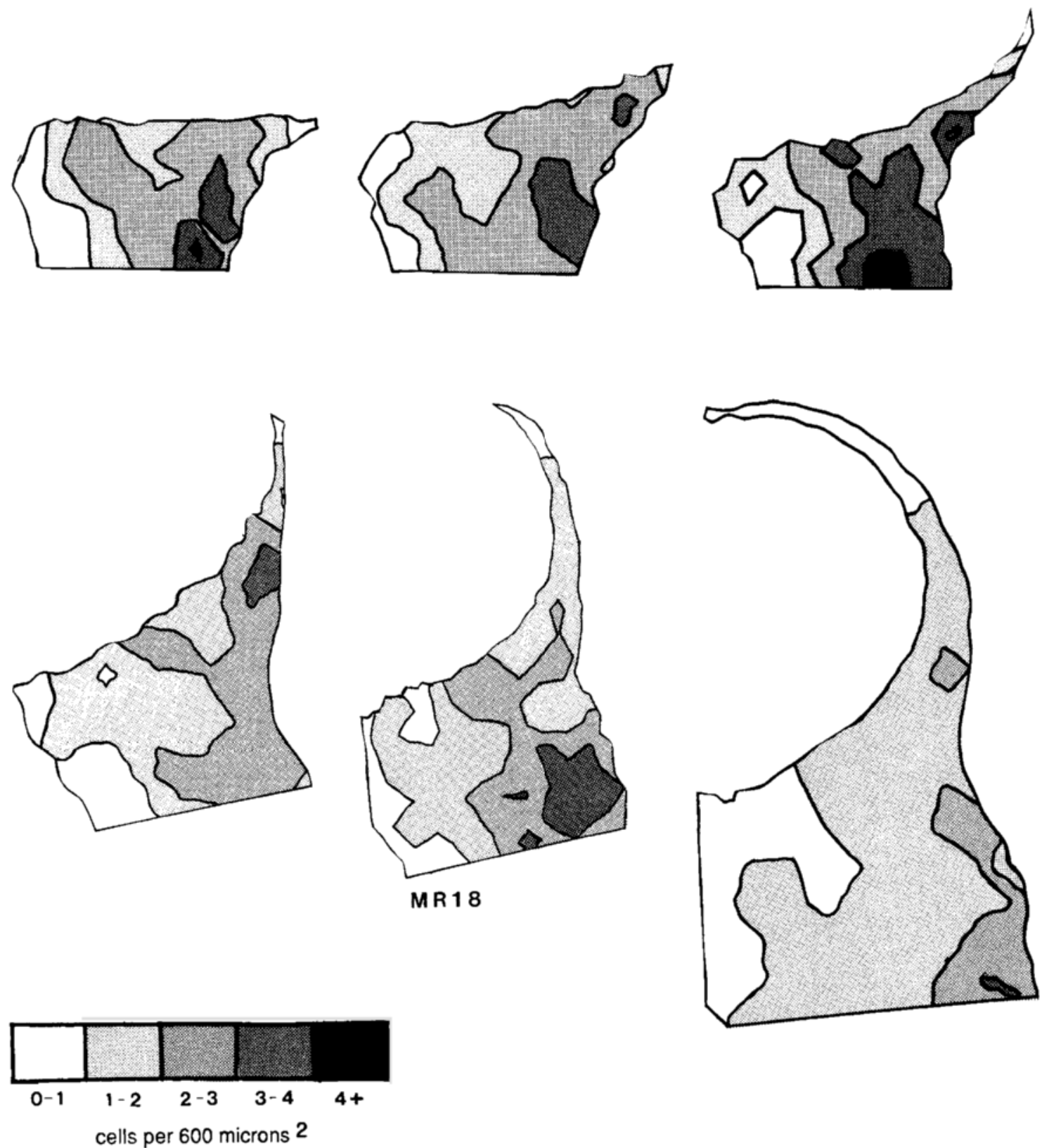

Fig. 3. Representative contour maps of mesenchymal cell density in the mesencephalic neural folds during their elevation. Contour maps were generated using a smoothed spatial averaging algorithm (see

Materials and Methods) and then were gray level coded as shown in the legend to allow better pattern discrimination. $\mathrm{m}$, medial; $\mathrm{l}$, lateral; d, dorsal. 

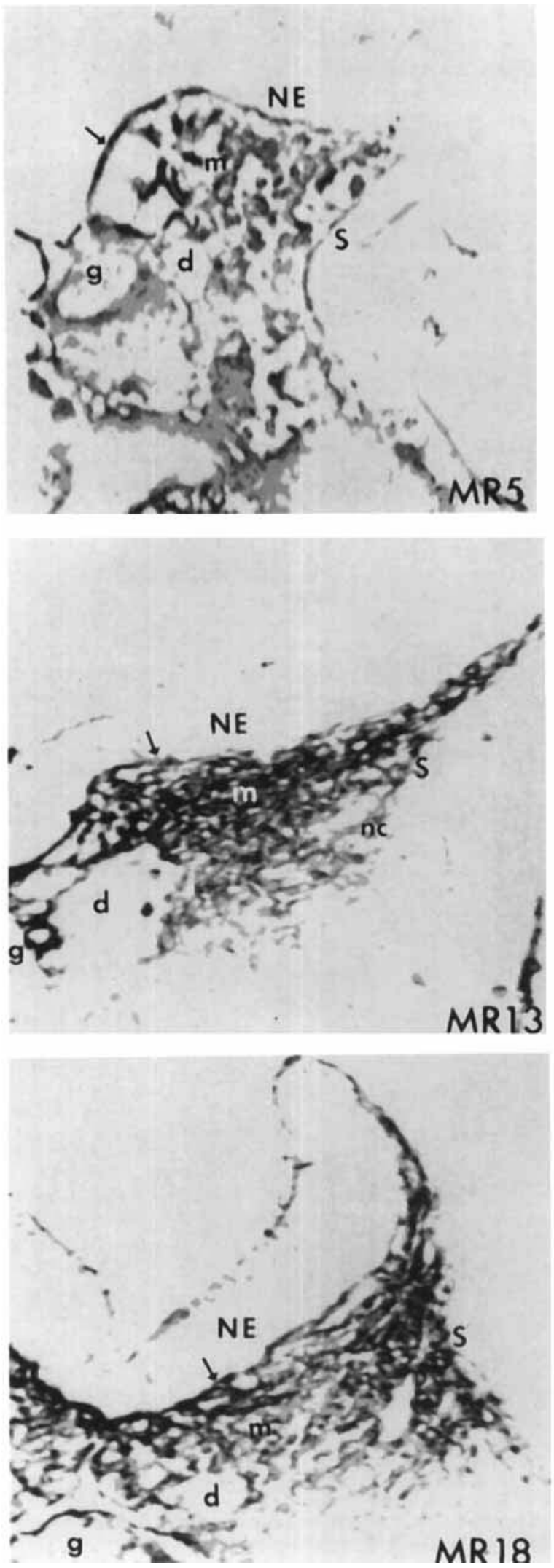
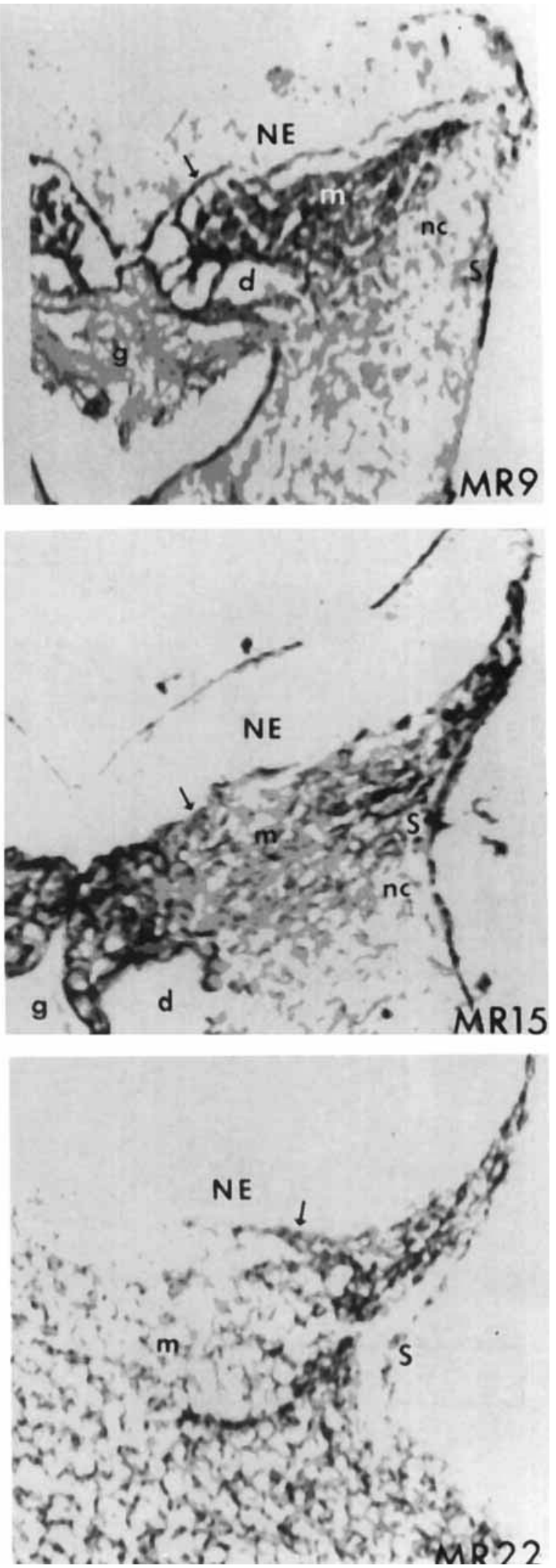
were digested with chrondroitinase $\mathrm{ABC}$ (ChABC). ChABC specifically digests chondroitin sulfates $\mathrm{A}, \mathrm{B}$, and $\mathrm{C}$ but also, even under ideal conditions, may extract HA (Derby and Pintar, 1978). The intensity of Alcian blue staining of ChABC-digested sections was highly variable between embryos of the same morphological rating and even between sections of the same embryo. Although the amount of Alcian blue-stained material observed in the ChABC-digested sections was usually less than that in difference picture images, the patterns were indistinguishable.

The images displayed in Figure 4 are representative of difference picture images from each stage studied. In the early stages of neural fold elevation (MR3-MR5) Alcian blue-stained HA is distributed throughout the mesenchymal compartment of the convex fold with increased concentrations found in the more medial mesenchymal regions adjacent to the rim of the neural groove. Staining often extends into the cell-free region adjacent to the neural groove. The neuroepithelial basement membrane $(\mathrm{NE} / \mathrm{Bm})$ is well stained from the midpoint of the neural groove extending onto its rim to about the midfold.

In MR7 and MR9 folds, the very lateral portions of the fold, those regions occupied by neural crest cells, are not stained. However, the relative amount of Alcian blue-stained HA is slightly increased in the medial and central regions of these folds. In both MR7 and MR9 folds the $\mathrm{NE} / \mathrm{Bm}$ is well-stained in the neural groove as at earlier stages.

The relative concentrations of HA in the mesenchymal compartment are increased in the subsequent stages as the folds begin to elevate (MR11-13). In these flattened and partially elevated folds, intense HA staining extends from the medial mesenchymal compartment enclosed by the neural groove and the dorsal aorta through the dorsal portions of the central fold and into the base of the narrowed dorsolateral edge of the elevating fold. The stain is excluded from the densely populated ventrolateral fold. As in earlier stages $\mathrm{NE} / \mathrm{Bm}$ is distinctly stained only in the more medial regions of the neural fold.

At MR15 the relative concentration of Streptomyces hyaluronidase-sensitive Alcian blue-stained material decreases in the medial and central regions of the fold, but increases in the dorsolateral fold under the elevating edge of the fold. As elevation continues, the increased area of expansion of the fold is accompanied by a shift in the pattern of Alcian blue staining (MR18). The lateral regions of the mesenchyme become the more heavily stained regions. By completion of eleva-

Fig. 4. Representative difference picture images (DPI) showing the location of hyaluronate within the mesenchyme of the mesencephalic neural folds during their elevation. 'The DPI's were produced by subtracting the image of an Alcian blue-stained, Streptomyces hyaluronidase-digested section from the image of a stained, undigested adjacent section. The resultant image is an accurate depiction of the location of Alcian blue-stained, Streptomyces hyaluronidase-sensitive material, or hyaluronate. The DPI's were further processed using a thresholding function and a median filter to segment the gray levels corresponding to relative hyaluronate concentrations into six steps and smooth the image to eliminate graininess. NE, neuroepithelium; $\mathrm{S}$, surface ectoderm; m, mesenchyme; nc, neural crest region; arrow, $\mathrm{NE} / \mathrm{Bm} ; \mathrm{g}$, gut; d, dorsal artery. $\times 163$. tion the medial mesenchymal region appears to be only diffusely stained (MR22). The NE/Bm is also very diffusely stained; only its lateral portions show any significant HA localization.

\section{Stages of Neural Fold Elevation}

Based on the preceding results, we have divided the process of neural fold elevation into five stages. Neural fold elevation is a dynamic process; the transition from one stage to another is not an abrupt event. Although we have associated specific morphological ratings with each stage, certain ratings span between stages, with some individuals possessing characteristics of a preceeding or subsequent stage. The stages of neural fold elevation are presented diagrammatically in Figure 5.

Stage 1 (CONVEX FOLD; MR3 to MR7) is a growth stage. It is characterized by a significant increase in fold cross-sectional area, and in total mesenchymal cell number. The expansion of the fold at MR7 is associated with a decrease in mesenchymal cell density in the medial and central fold regions and an overall increase in HA concentration.

Stage 2 (PLANAR FOLD; MR7 to MR9) is characterized by changes in neuroepithelial organization. The neuroepithelium assumes a pseudostratified organization concomitant with the flattening of the folds. No significant changes in mesenchymal cell or HA distribution occur.

Stage 3 (INITIAL ELEVATION; MR11 to MR13) is characterized by both the reorganizationof the mesenchyme and the neuroepithelium. Mesenchymal cell density is significantly increased in all fold regions as is the amount of Alcian blue-stained HA. Mesenchymal cells are observed to be oriented in parallel to the transverse plane of the fold. The fold length and basement membrane length increases significantly during this stage and the acquisition of the lateral furrow is reflected in a decrease in the fold length to basement membrane length ratio and a decrease in interfold distance.

Stage 4 (MESENCHYMAL EXPANSION; MR15) During this stage there is no change in the neuroepithelial parameters, fold length and basement membrane length, but there is a decrease in interfold distance and interfold angle. There is a significant increase in mesenchymal cross-sectional area, but no increase in the lateral dimension of the fold. In the central and medial fold, mesenchymal cell density decreases and the parallel stacking of mesenchymal cells is not observed although it remains characteristic of the more lateral fold. The relative amount of Alcian blue-stained HA decreases in the central fold and increases in the lateral fold.

Stage 5 (FOLD CONVERGENCE; MR18 to FOLD CLOSURE) is characterized by significant changes in both neuroepithelial and mesenchymal parameters. There is a significant decrease in interfold distance with a concomitant increase in basement membrane length and in the curvature of the fold (fold length to basement membrane length ratio), but there is no change in interfold angle. There is a significant increase in mesenchymal cross-sectional area, but no increase in the lateral dimension of the fold. Mesenchymal expansion is directed in a dorsoventral plane as 

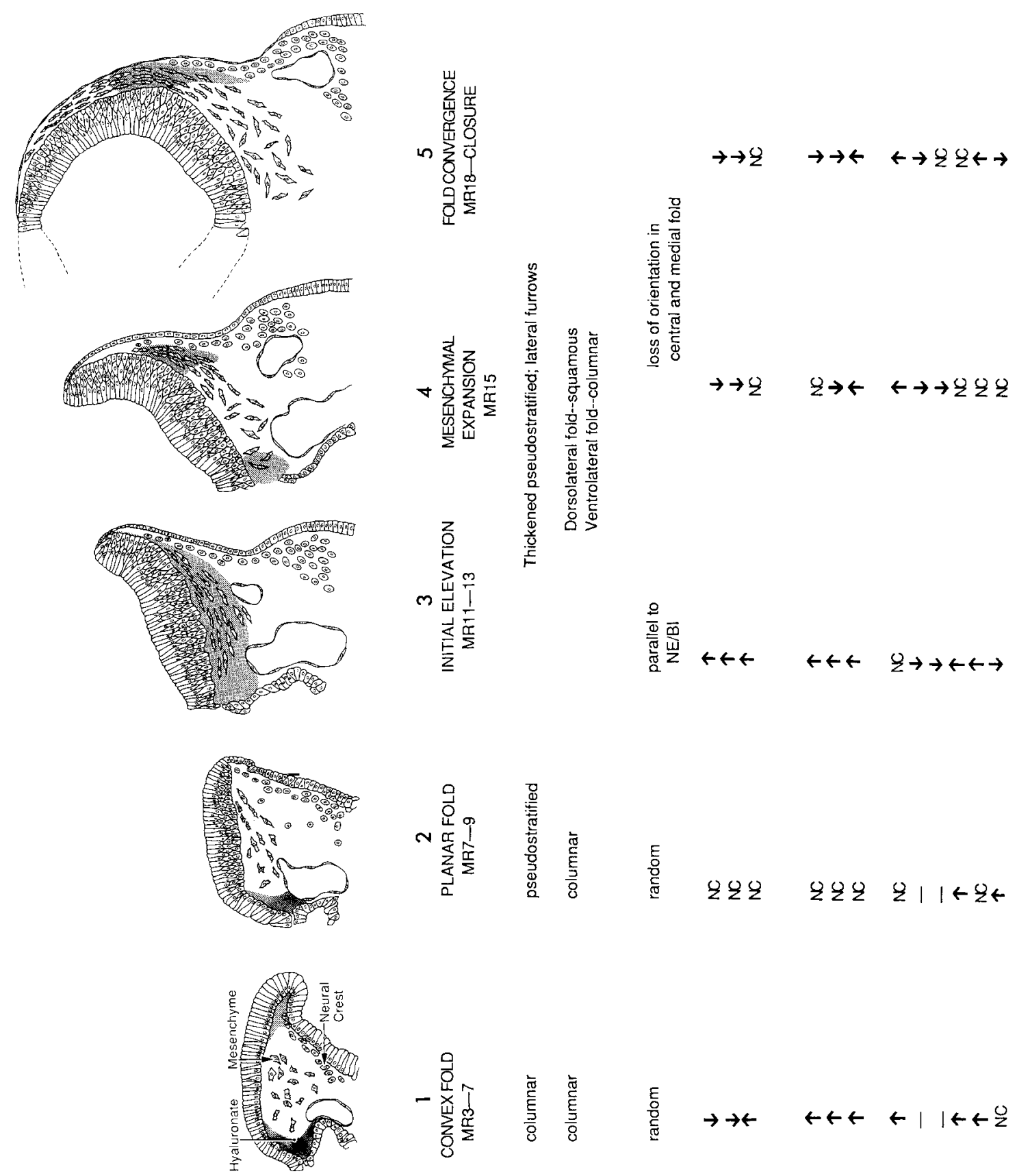

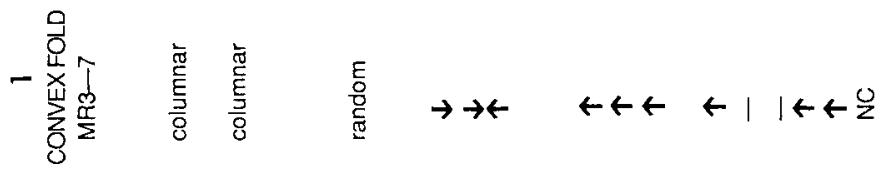

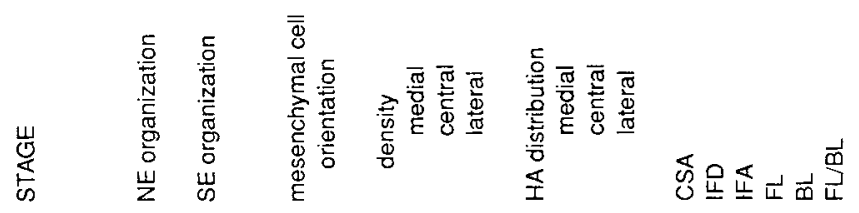

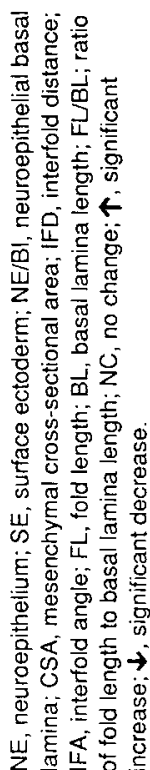

Fig. 5. Diagrammatic representation of the stages of neural fold elevation and associated changes in mesenchymal, neuroepithelial, and surface ectodermal organization. 
evidenced in the separation between the neural groove and the foregut or notochord. Mesenchymal cell density decreases in the central and medial fold regions as does the relative amount of Alcian blue-stained HA. Mesenchymal cell orientation in the fold appears random.

\section{DISCUSSION}

The results of this study have allowed us to identify five distinct stages in the elevation and closure of the mouse mesencephalic neural folds. The conversion of one stage to another is associated with quantifiable changes in fold shape and degree of elevation, as well as with histologically observable changes in the organization of the neuroepithelium, surface ectoderm, and the mesenchyme. Interstage transition is also associated with distinct temporal and spatial patterns of mesenchymal cell and hyaluronate (HA) distribution. By examining the contributions of the neuroepithelium, surface ectoderm, and mesenchyme to the changes in fold shape and degree of elevation that are associated with the conversion of one stage to another, we have been able to reflect on the role of these major fold components in neural fold elevation. The successful closure of the cranial neural folds to form a neural tube may involve two distinct, but interdependent, processes: neural fold elevation in which the folds are pushed medially towards the midline, decreasing the interfold angle; and neural fold convergence in which the mediad curvature of the folds is increased, placing the folds in apposition to allow fusion. Our results suggest that mesenchymal reorganization plays a major role in fold elevation, whereas the mediad convergence of the folds is mainly the result of neuroepithelial and surface ectodermal reorganization.

\section{Mesenchymal Reorganization in Initial Elevation}

The conversion of a stage 2 planar to a stage $3 \mathrm{~V}$ shaped fold is accompanied by significant mesenchymal reorganization. Mesenchymal cell density and HA concentration increase and mesenchymal cell orientation becomes distinctly polarized; i.e., mesenchymal cells become oriented parallel to the neuroepithelial basement membrane $(\mathrm{NE} / \mathrm{Bm})$. This polarization of the mesenchyme may be a consequence of the significant lateral expansion of the neuroepithelium which occurs at stage 3 , as indicated by the increase in its basement membrane length (BL, Table 1a). The significant increase in mesenchymal cell and HA density at stage 3 would be likely to result in increased cell-cell and cellmatrix interactions, thus increasing the ridigity of the mesenchyme and limiting its ability to expand. The lateral growth of the neuroepithelium would be expected to put some degree of tension on the now more rigid underlying mesenchyme. In vitro fibroblasts are observed to orient themselves in response to tension placed on their surrounding extracellular matrix (ECM) (Stopak and Harris, 1982; Lewis, 1984). Similarly, the lateral expansion of the neuroepithelial plate may cause the ECM and enmeshed cells to be oriented in the direction of this expansion (Fig. 2).

The initial elevation of the folds to a V-shape at stage 3 may be a consequence of constraints placed on neuroepithelial lateral expansion by the remodelling mesenchyme. The lateral pull of the expanding neuroepithelium would eventually be counteracted by the resistance or medial pull of the increasingly more rigid mesenchyme. The resultant force generated by the sum of these two forces would be dorsally directed and would elevate the folds to produce the characteristic stage $3 \mathrm{~V}$-shape. Other investigators (Morriss and New, 1979; Jacobson and Tam, 1982) have proposed that this initial elevation of the cranial neural folds is primarily the result of a longitudinal or rostrocaudal pull produced by an increase in the cervical flexure. We propose that the necessary forces may be generated locally, by the remodelling of the mesenchyme and neuroepithelium, and are not dependent on the elongation of the neural plate as a whole.

\section{Mesenchymal Expansion in Neural Fold Elevation}

The elevation of the neural folds at stage 4 is associated with a significant increase in mesenchymal cross-sectional area and with distinct changes in patterns of mesenchymal cell and HA distribution. No significant changes are observed in the degree of curvature of the neural plate. The analysis of patterns of HA distribution in difference picture images of stage 4 neural folds reveals that the relative concentration of $\mathrm{Al}$ cian blue-stained HA is decreased in the central fold (Fig. 4). This decrease in HA content may be a consequence of the observed expansion of the fold mesenchyme at stage 4 which would be accompanied by a separation of HA Alcian blue-binding sites and a decrease in Alcian blue staining. Such expansion would also be expected to result in the dispersal or separation of resident mesenchymal cells, causing a decrease in the cell density in the central fold. In fact, such a decrease is seen in plots of smoothed spatial averages (Fig. 3).

At concentrations observed in embryonic tissues, HA molecules intermesh with each other and with other ECM components, entrapping water and ions and thus causing an increased hydration of the tissue (Comper and Laurent, 1978). This increased hydration is accompanied by an increase in tissue volume, unless the expansion of the HA-rich extracellular matrix (ECM) is restricted by cell-cell or cell-matrix interactions. If such interactions are subsequently disrupted, the tissue will experience a sudden expansion. Such a model has been proposed by Toole (1981) to explain the sudden expansion of extracellular spaces which precedes corneal, cardiac cushion, sclerotome, and neural crest cell migration. We propose that the expansion of the central mesenchyme observed in stage 4 folds is the result of the reorganization of an HA-rich ECM. As HA is newly synthesized in the mesenchyme, its expansion may be held in check by mesenchymal cell-cell interactions. When these interactions are disrupted in the central mesenchyme, possibly due to the tension placed on the mesenchyme by the lateral growth of the neuroepithelium, the HA-rich matrix is allowed to expand, increasing the cross-sectional area of the fold mesenchyme and separating resident mesenchymal cells. At stage 4 the neural groove is firmly attached to the subjacent notochord and endoderm by strands of ECM (Fig. 4; Tuckett and Morriss-Kay, 1986). Thus any expansion of the central mesenchyme would cause the folds to move medially towards one another, decreasing the interfold angle and elevating the folds. This mediad elevation may be crucial for successful neural tube clo- 
sure. The mediad bending of the dorsolateral fold, effected by the formation of lateral furrows in the neuroepithelium, may not be sufficient to ensure that the folds make contact.

From this model one would predict that any perturbation of the synthesis or molecular organization of $\mathrm{HA}$ in the fold mesenchyme would result in a failure of the folds to elevate. The results of our own studies in which mouse embryos were cultured in diazo-oxo-norleucine (DON), an agent which blocks glucosamine formation and thus glycosaminoglycan synthesis (Ghosh et al., 1960; Telser et al., 1965), provide support for this model (Morris-Wiman and Brinkley, 1990a,b). The cranial neural folds of these embryos do not elevate, but rather appear blocked in the planar stage or, after acquiring a slight mediad curvature, are reminiscent of stage 3 embryos. The results of another recent study would seem to dispute the role of $\mathrm{HA}$ in the elevation of the cranial neural folds. To determine whether normal neurulation occurs when HA is depleted, Morriss-Kay, Tuckett, and Solursh (1986) incubated rat embryos in the enzyme Streptomyces hyaluronidase $(\mathrm{SH})$ to degrade HA specifically. Neural fold elevation was retarded but not prevented. However, the effects of SH treatment, confirmed by a decrease in Alcian blue staining and a decrease in extracellular space, were not apparent in the central mesenchyme until after $16 \mathrm{hr}$ of culture. If the development of embryos in culture was indistinguishable from that in vivo during this period, as was stated by the investigators, our results indicate that the important events in fold elevation involving mesenchymal expansion would have already occurred by $16 \mathrm{hr}$ of culture.

The last stage of neural tube formation, convergence, is also characterized by a significant expansion of the fold mesenchyme, but this expansion occurs in the dorsolateral dimension as there is no increase in the lateral dimension of the fold (Table 1b). Lateral expansion of the fold may be prohibited by the organization of the mesenchyme; i.e., the orientation of mesenchymal cells in the transverse plane may preclude expansion in this direction. Thus with the disruption of the attachment of the neural groove to the foregut and notochord, expansion of the central and medial mesenchyme results in pure dorsoventral expansion (Fig. 2) with no change in the interfold angle (Table 1b). A decrease in Alcian blue-stained HA in the central and medial fold is comcomitant with this dorsoventral expansion, suggesting that HA may play a major role. Such a decrease in $\mathrm{HA}$ concentration was also observed in a study by McLone and Knepper (1986) at the completion of neural fold closure. The results of Morriss-Kay et al. (1986) also suggest that the reorganization of an HA-rich ECM is resonsible for this mesenchymal expansion. The incubation of rat embryos with Streptomyces hyaluronidase for $48 \mathrm{hr}$ blocked the dorsoventral expansion of the fold.

\section{Lateral Furrow Formation in Neural Fold Convergence}

The successful closure of the neural folds depends not only on the directed expansion of the mesenchyme to elevate the folds, but also on an increase in the mediad curvature of the fold to allow convergence of the fold edges. In other species studied, the increase in the fold's mediad curvature is preceded by the formation of lateral constrictions or furrows in the neuroepithelium
(chick-Schoenwolf and Fisher, 1983; salamander-Brun and Garson, 1983; frog-Schroeder, 1970). The results of our morphological observations indicate that this is also the case in the cranial neural folds of the mouse (Fig. 2). The formation of a furrow in the neuroepithelium would serve to bend the dorsolateral fold edge medially. Thus any subsequent lateral elongation or growth of the neuroepithelium, such as that observed at stage 5 , would be directed medially and insure fold convergence. The formation of lateral furrows may be the result of localized neuroepithelial cell-shape changes (Schoenwolf and Franks, 1984) or produced by the mediad growth of the surface ectoderm, bending the neural plate (Schoenwolf and Franks, 1984; Brun and Garson, 1983).

The location of the lateral furrows may be dictated by the subjacent basement membrane and mesenchyme. In this study (Fig. 4) and in a previous study by Morriss and Solursh (1978a), the NE/Bm was observed to stain intensely in the medial regions of the fold, but binds Alcian blue only faintly in the region subjacent to the furrow. The importance of the basement membrane in the determination of cell shape and behavior has been well-documented (for review, see Bernfield et al., 1984). In developing salivary glands, the epithelium composing the distal bulb of the gland undergoing morphogenetic changes is associated with less Alcian blue-staining and a greater turnover of ${ }^{3} \mathrm{H}$-glucosamine than the epithelium composing the stalk region which has assumed a stable morphology (Bernfield et al., 1972; Bernfield and Banerjee, 1982). The lack of staining in the more lateral regions of the neural fold may be indicative of an increased turnover of HA, contributing to an increased plasticity of the morphology of the neuroepithelium in this region. This increased plasticity may permit furrow production. The prominently stained basement membrane of more medial regions may stabilize the neuroepithelium and preclude neuroepithelial cell shape change. The furrows form over a region of the dorsolateral mesenchyme with an increased cell density (Fig. 2). The formation of the furrows is also preceded by an increase in the density of HA in the dorsolateral fold base (Fig. 4). This increase in mesenchymal cell and HA density may be necessary to provide support for, or buttress, the curving dorsolateral fold.

\section{ACKNOWLEDGEMENTS}

The work presented in this manuscript was done in partial fulfillment of the doctoral degree in Anatomy and Cell Biology at the University of Michigan.

\section{LITERATURE CITED}

Bernfield, M.R., and S.D. Banerjee 1982 The turnover of basal lamina glycosaminoglycan correlates with epithelial morphogenesis. Dev. Biol., 90:291-305.

Bernfield, M.R., S.D. Banerjee, and R.H. Cohn, 1972 Acid mucopolysaccharides (glycosaminoglycans) at the epithelial-mesenchymal interface of mouse embryo salivary glands. J. Cell Biol., $52: 664-673$.

Bernfield, M.R., S.D. Banerjee, J.E. Koda, and A.L. Rapraeger, 1984 Remodeling of the basement membrane as a mechanism of morphogenetic tissue interaction. In: The Role of Extracellular Matrix in Development. R.L. Trelstad, ed. Alan R. Liss, Inc., New York, pp. 545-572.

Brinkley, L.L., and F.L. Bookstein, 1986 Cell distribution during 
mouse secondary palate closure. II. Mesenchymal cells. J. Embryol. Exp. Morphol., 96:111-130.

Brinkley, L.L., and J. Morris-Wiman, 1987a Computer-assisted analysis of hyaluronate distribution during morphogenesis of the mouse secondary palate. Development, 100:629-635.

Brinkley, L.L., and J. Morris-Wiman, 1987b Effects of chlorcyclizineinduced glycosaminoglycan alterations on patterns of hyaluronate distribution during morphogenesis of the mouse secondary palate. Development, 100:637-640.

Brown, N.A., and S. Fabrio 1981 Quantitation of rat embryonic development In Vitro: A morphological scoring system. Teratology, 24(1):65-78.

Brun, R.B., and J.A. Garson 1983 Neurulation in the Mexican salamander (Amblystoma mexicanum): A drug study and cell shape analysis of the epidermis and the neural plate. J. Embryol. Exp. Morphol., 74:275-295.

Connelly, T.G., and F.L. Bookstein 1983 Method for 3-dimensional analysis of patterns of thymidine labelling in regenerating and developing limbs. In: Limb Development and Regeneration, Part A. J.F. Fallon and A.I. Caplan, ed. Alan R. Liss, Inc., New York, pp. $525-536$.

Comper, W.D., and T.C. Laurent 1978 Physiological function of connective tissue polysaccharides. Physiol. Rev., 58:255-315.

Derby, M.A., and J.E. Pintar 1978 The histochemical specificity of Streptomyces hyaluronidase and chondroitinase ABC. Histochem. J., $10: 529-547$.

Fisher, M., and M. Solursh 1977 Glycosaminoglycan localization and role in maintenance of tissue spaces in early chick embryos. $J$ Embryol. Exp. Morphol., 42:195-207.

Ghosh, S., H.J. Blumenthal, E.A. Davidson, and S. Roseman 1960 Glucosamine metabolism, V. Enzymatic synthesis of glucosamine-6-phosphate. J. Biol. Chem., 235:1265-1272.

Heifetz, A., W.J. Lennarz, B. Libbus, Y Hsu 1980 Synthesis of glycoconjugates during the development of mouse embryos in vitro. Dev. Biol., 80:398-408.

Karfunkel, P. 1974 The mechanism of neural tube formation. Int. Rev. Cytol., 38:245-271.

Knoll, T.F., L.L. Brinkley, and E.J. Delp 1985 Difference pictures algorithms for the analysis of extracellular components of histological images. J. Histochem. Cytochem., 33:261-267.

Lewis, J. 1984 Morphogenesis by fibroblast traction. Nature, 307(5950):413.

McLone, D.G., and P.A. Knepper 1986 Role of complex carbohydrates and neurulation. Ped. Neurosci., 12:2-9.
Morriss, G.M. and M. Solursh 1978a Regional differences in mesenchymal cell morphology and glycosaminoglycans in early neuralfold stage rat embryos. J. Embryol. Exp. Morphol., 46:37-52.

Morriss, G.M. and M. Solursh 1978b The role of the primary mesenchyme in normal and abnormal morphogenesis of mammalian neural folds. Zoon, $6: 33-38$

Morriss-Kay, G.M. 1981 Growth and development of pattern in the cranial neural epithelium of rat embryos during neurulation. J. Embryol. Exp. Morhpol., 65:225-241.

Morriss-Kay, G.M., F. Tuckett and M. Solursh 1986 The effects of Streptomyces hyaluronidase on tissue organization and cell cycle time in rat embryos. J. Embryol. Exp. Morphol., 98:59-70.

Morris-Wiman, J., and L.L. Brinkley 1990a The role of the mesenchyme in mouse neural fold elevation. I. Patterns of mesenchymal cell proliferation in embryos developing in vitro. Am. J. Anat. (Submitted).

Morris-Wiman, J., and L.L. Brinkley 1990b The role of the mesenchyme in mouse neural fold elevation. II. Patterns of hyaluronate synthesis and distribution in embryos developing in vitro. Am. J. Anat. (Submitted).

Ohya, T., and Y. Kaneko 1970 Novel hyaluronidase from Streptomyces. Biochim. Biophys. Acta, 198:607-609.

Schoenwolf, G.C., and M. Fisher 1983 Analysis of the effects of Streptomyces hyaluronidase on the formation of the neural tube. J. Embryol. Exp. Morphol., 73:1-15.

Schoenwolf, G.C., and M.V. Franks 1984 Quantitative analyses of changes in cell shapes during bending of the avian neural plate. Dev. Biol., 105:257-272.

Solursh, M., and G.M. Morriss 1977 Glycosaminoglycan synthesis in rat embryos during the formation of the primary mesenchyme and neural folds. Dev. Biol., 57:75-86.

Stopak, D., and A.K. Harris 1982 Connective tissue morphogenesis of fibroblast traction. Dev. Biol., 90(2):383-398.

Telser, A., H.C. Robinson, and A. Dorfman 1965 The biosynthesis of chondroitin-sulfate protein complex. Proc. Nat. Acad. Sci. USA, 54:912-919.

Toole, B.P. 1981 Glycosaminoglycans in morphogenesis. In: Cell Biology of Extracellular Matrix. E. Hay, ed. Plenum Press, New York, pp. 259-294.

Tuckett, F., and Morriss-Kay 1986 The distribution of fibronectin, laminin and entactin in the neurulating rat embryo studied by indirect immunof luorescence. J. Embryol. Exp. Morphol., 94:95112. 